\title{
An Asian Problem, Oligosaccharides in Cow's Milk Formula (CMF) Causing Anaphylaxis in Older Children, 2 Years Above and Prenatal and Post Natal Mothers in Singapore
}

\section{Yoke Chin Giam*}

Senior Consultant Pediatric Dermatologist, National Skin Centre, Singapore

*Corresponding author: Yoke Chin Giam, Senior Consultant Pediatric Dermatologist, National Skin Centre, 1 Mandalay Road, Singapore, E-mail: ycgiam@nsc.com.sg Received date: June 06, 2017; Accepted date: June 21, 2017; Published date: June 29, 2017

Copyright: @ 2017 Giam YC, et al. This is an open-access article distributed under the terms of the Creative Commons Attribution License, which permits unrestricted use, distribution, and reproduction in any medium, provided the original author and source are credited.

\section{Summary}

I had been looking at Asian-only problems, and found this interesting, an "Oligosaccharides in milk causing anaphylaxis in some children and mothers" when oligosaccharide is safe in many other countries.

Short chain Galacto-oligosaccharides (scGOS) or prebiotic is a carbohydrate, is made of short sugar chains limited to to seven or less in length, synthesized by B galactosidases [1]. Prebiotics in infants are suggested to have effects, as in animal studies [2] an impact on certain immune markers, and diminished allergic sensitization on young female mice and reduced allergic sensitivities like allergic asthma.

Prebiotics are added as supplements into infant formula to boost the immune status of the children. It was unusual to develop anaphylaxis in this relatively safe-approved infant formula, and that these reactions can appear in children and adults, occurring mainly in South East Asia.

A number of GOS-structures have been found, concentrated in human colostrum, not in foods. This prebiotic has a safe history of use, and has a US FDA-approved Generally Recognized as Safe (GRAS) status. Besides being added in infant milk formulae worldwide in Americas, Europe, Australia, and Asia), in recent years, it is added to food products like food supplements and yoghurt.

However, reports of anaphylaxis have been reported in South East Asia, Vietnam [3] and Japan. In Singapore, this has affected preschool children and post natal mothers. These children were above 2-4 years in age, had an atopic background, no food allergy, and tolerant of milk. All had a similar clinical presentation of symptoms within $30 \mathrm{~min}$ of ingestion, suggesting an IgE -mediated immediate allergy. This has been well discussed and investigated by Chiang [4].

The Skin Prick tests were done to scGOS, patients' CMF [4] without scGOS, and long chain lc GOS [4] and the investigations point to the conclusion of an IgE-mediated mechanism, on the data showing these positive results to scGOS, and not to the other formula without scGOS supplementation [4]. The Activation of the basophil activation (BAT) markers also showed dose-related responses to the milk formula supplemented with scGOS, scGOS alone, implicating scGOS as the allergen [4]. All children and mothers affected [4,5], demonstrated positively, by the skin prick test to scGOS and those subjects tested to BAT, were positive for scGOS. However, it was thought that the primary sensitization was probably by another allergen though these tests were positive to the low [4] prebiotic scGOS. Soh, in his report on the 4 post natal lactating mothers [5] confirmed the positive skin prick tests and positive basophil activation tests to sc-oligosaccharides. This reaction is indeed rare. Surveillance at the Emergency departments had identified the above patients.

In recent times, from 2012, this scGOS have been removed from many of the formulations of milk formula in Singapore, produced for infants and lactating women. As these, now, do not contain scGOS, anaphylactic reactions to scGOS can be prevented in both. There have been nine cases reported so far [4,5]. This geographic presence of these cases of GOS-related allergy to South East Asia is unusual. It suggests that the primary sensitizer is likewise, specific to South East Asia. It may be caused by a mosquito bite. The mechanism of sensitization remains unclear. A plausible explanation is that the individual may become sensitized to the oligosaccharide when it happens to bind to a carrier protein, but further tests are required [6-8].

This reaction is rare. Surveillance at the Emergency departments had identified the above patients.

Further studies are now conducted on LGOS and BGOS, which are variants of GOS, on adult candidates, allergic to scGOS, in Singapore.

\section{Acknowledgement}

Permission was granted by Dr. Soh JY to highlight this unusual presentation as an Asian phenomenon.

\section{References}

1. Coulier L, Timmermans J, Van Den DR, Bas R, Haaksman I, et al. (2009) In depth characterisation of prebiotic galacto-oligosaccharides by a combination of analytical techniques. J Agric Food Chem 57: 8488-8495.

2. Moro G, Minoli I, Mosca M, Fanaro S, Jelinek J, et al. (2002) Dosage related bifidogenic effects of galacto and fructo oligosaccharides in formula fed term infants. J Ped gastroenterol Nutr 34: 291-295.

3. Vo TH, Le NH, Patel MS, Phan LT, Tran Mihn LL, et al. (2012) Acute allergic reaction in Vietnamese children after drinking a new milk product. Foodbourne Pathog Dis 9: 156-159.

4. Chiang WC, Huang CH, Llanora GV, Gerez I, Goh SH, et al. (2012) Anaphylaxis to Cow's milk formula containing short-chain galactooligosaccharide. J Allergy Clin Immunol 130: 1361-1367.

5. Soh JY, Chiang WC, Huang CH, Woo CK, Ibrahim I, et al. (2017) An unusual cause of food-induced anaphylaxis in mothers. World Allergy Organ J 10: 3 .

6. Calamari AM, Poppa M, Villalta D, Pravettoni V (2015) Alpha-gal anaphylaxis: the first case report in Italy. Eur Ann Allergy Clin Immunol 47:161-162.

7. Chinuki Y, Ishiwata K, Yamaji K, Takahashi H, Morita E, et al. (2016) Haemaphysalis longicornis tick bites are a possible cause of red meat allergy in Japan. Allergy 71: 421-425. 
Citation: Giam YC (2017) An Asian Problem, Oligosaccharides in Cow's Milk Formula (CMF) Causing Anaphylaxis in Older Children, 2 Years Above and Prenatal and Post Natal Mothers in Singapore. J Allergy Ther 8: 260. doi:10.4172/2155-6121.1000260

Page 2 of 2

8. Commins SP, Jerath MR, Cox K, Erickson LD, Platts-Mills T, et al. (2016)

Delayed anaphylaxis to alpha-gal, an oligosaccharide in mammalian meat. Allergol Int 65: 16-20. 